\title{
Research Article \\ $H^{1} \cap L^{p}$ versus $C^{1}$ Local Minimizers
}

\author{
Yansheng Zhong \\ Department of Mathematics, Fujian Normal University, Fuzhou 350117, China \\ Correspondence should be addressed to Yansheng Zhong; zhyansheng08@163.com
}

Received 18 March 2014; Accepted 9 April 2014; Published 28 April 2014

Academic Editor: Jianqing Chen

Copyright (c) 2014 Yansheng Zhong. This is an open access article distributed under the Creative Commons Attribution License, which permits unrestricted use, distribution, and reproduction in any medium, provided the original work is properly cited.

We show that a local minimizer of $\Phi$ in the $C^{1}$ topology must be a local minimizer in the $H^{1} \cap L^{p}$ topology, under suitable assumptions for the functional $\Phi=(1 / 2) \int_{\Omega}|\nabla u|^{2}+(1 / p) \int_{\Omega}|u|^{p}-\int_{\Omega} F(x, u)$ with supercritical exponent $p>2^{*}=2 n /(n-2)$. This result can be used to establish a solution to the corresponding equation admitting sub- and supersolution. Hence, we extend the conclusion proved by Brezis and Nirenberg (1993), the subcritical and critical case.

\section{Main Results for Supercritical Exponent}

We consider the following functional:

$$
\Phi=\frac{1}{2} \int_{\Omega}|\nabla u|^{2}+\frac{1}{p} \int_{\Omega}|u|^{p}-\int_{\Omega} F(x, u),
$$

where $\Omega \subset \mathbb{R}^{n}$ with smooth boundary, supercritical exponent $p>2^{*}=2 n /(n-2)$, and $F(x, u)=\int_{0}^{u} f(x, s) d s$ satisfies the growth condition:

$$
|f(x, u)| \leqslant C\left(1+|u|^{\ell}\right) \quad \text { with } \ell<p,
$$

as well as the usual assumptions that $f$ is measurable in $x$ and continuous in $u$.

Our main results are the following.

Theorem 1. Assuming that $u_{0} \in H_{0}^{1}(\Omega) \cap L^{p}(\Omega)$ is a local minimizer of $\Phi$ in the $C^{1}$ topology, there is some $r>0$, such that

$$
\Phi\left(u_{0}\right) \leqslant \Phi\left(u_{0}+v\right), \quad \forall v \in C_{0}^{1}(\bar{\Omega}) \quad \text { with }\|v\|_{C^{1}} \leqslant r .
$$

Then $u_{0}$ is also a local minimizer of $\Phi$ in the $H_{0}^{1}(\Omega) \cap L^{p}(\Omega)$ topology; that is, there exists $\epsilon_{0}>0$, such that

$$
\begin{array}{r}
\Phi\left(u_{0}\right) \leqslant \Phi\left(u_{0}+v\right), \quad \forall v \in H_{0}^{1}(\Omega) \cap L^{p}(\Omega) \\
\text { with }\|v\|_{H_{0}^{1}(\Omega) \cap L^{p}(\Omega)} \leqslant r,
\end{array}
$$

where the topology $X \triangleq H_{0}^{1}(\Omega) \cap L^{p}(\Omega)$ given by $\|\cdot\|_{X}=$ $\|\cdot\|_{H_{0}^{1}(\Omega)}+\|\cdot\|_{L^{p}(\Omega)}$.

It will be noted that an $X$ neighbourhood is much bigger than a $C_{0}^{1}$ neighbourhood. The proof depends on the special structure of $\Phi$, and the claim clearly would be false for a general function $\Phi$.

In order to prove this theorem, the following preparatory steps are critical. We begin with a theorem concerning the topology of $X$.

Theorem 2. Let $X$ be defined as in the above theorem; then $X$ is a reflexive and strictly convex Banach space with the duality $X^{*} \subset H^{-1}(\Omega) \oplus L^{q}(\Omega)((1 / p)+(1 / q)=1)$.

Proof of Theorem 2. Now we give a detailed proof for the reader's convenience.

At first we show that the definition of $\|\cdot\|_{X}$ is actually a norm. Obviously, separate points are as follows: if $\|x\|_{X}=0$, that is, $\|x\|_{H_{0}^{1}(\Omega)}+\|x\|_{L^{p}(\Omega)}=0$, then $x=0$. And positive homogeneity is $\|\alpha x\|_{X}=\|\alpha x\|_{H_{0}^{1}(\Omega)}+\|\alpha x\|_{L^{p}(\Omega)}=$ $|\alpha|\left[\|x\|_{H_{0}^{1}(\Omega)}+\|x\|_{L^{p}(\Omega)}\right]=|\alpha|\|x\|_{X}$. The triangle inequality is, for any $x, y \in X$,

$$
\begin{aligned}
\|x+y\|_{X}= & \|x+y\|_{H_{0}^{1}(\Omega)}+\|x+y\|_{L^{p}(\Omega)} \\
\leqslant & {\left[\|x\|_{H_{0}^{1}(\Omega)}+\|x\|_{L^{p}(\Omega)}\right] } \\
& +\left[\|y\|_{H_{0}^{1}(\Omega)}+\|y\|_{L^{p}(\Omega)}\right]=\|x\|_{X}+\|y\|_{X} .
\end{aligned}
$$


And then it shows that the space $X$ is complete; that is to say, any cauchy sequence $\left\{u_{n}\right\}$ in $\|\cdot\|_{X}$ will converge. From the definition of the norm $\|\cdot\|_{X}$, we know that $u_{n}$ is also the cauchy sequence in $H_{0}^{1}(\Omega)$ and $L^{p}(\Omega)$. By the completion of the Banach space $H_{0}^{1}(\Omega)$ and $L^{p}(\Omega)$, we know that $u_{n}$ will converge to $u_{1}$ in $H_{0}^{1}(\Omega)$ and $u_{n}$ will converge to $u_{2}$ in $L^{p}(\Omega)$. And, since $H_{0}^{1}(\Omega) \hookrightarrow L^{2}(\Omega)$, we know that $u_{n} \rightarrow u_{1}$ in $L^{2}(\Omega)$ and also, due to $L^{p}(\Omega) \subseteq L^{2}(\Omega)$, we also know that $u_{n} \rightarrow u_{2}$ in $L^{2}(\Omega)$, and, based on the uniqueness of the limit in $L^{2}(\Omega)$, we have $u_{1}=u_{2}$ (denoted by $u$ ). With this result, we have $u_{n}$ converge to $u$ in $X$, which implies that $X$ is complete. Thus, $X$ is a Banach space.

For strictly convex, which is based on the definition of the strictly convex of Banach space, we need to show that if $x \neq y$ and $\|x\|_{H_{0}^{1}(\Omega)}+\|x\|_{L^{p}(\Omega)}=\|y\|_{H_{0}^{1}(\Omega)}+\|y\|_{L^{p}(\Omega)}=1$, then $\|x+y\|_{H_{0}^{1}(\Omega)}+\|x+y\|_{L^{p}(\Omega)}<2$, which can be done by the following inequality:

$$
\begin{aligned}
\|x+y\|_{H_{0}^{1}(\Omega)}+\|x+y\|_{L^{p}(\Omega)} \\
\quad \leqslant\left(\|x\|_{H_{0}^{1}(\Omega)}+\|y\|_{H_{0}^{1}(\Omega)}\right)+\left(\|x\|_{L^{p}(\Omega)}+\|y\|_{L^{p}(\Omega)}\right) \\
\quad=\left(\|x\|_{H_{0}^{1}(\Omega)}+\|x\|_{L^{p}(\Omega)}\right)+\left(\|y\|_{H_{0}^{1}(\Omega)}+\|y\|_{L^{p}(\Omega)}\right) \\
\quad=2 .
\end{aligned}
$$

And the fact " $=$ " in (6) is true if and only if $x=c y$ with the constant $c>0$ in consequence of the uniformly convex space $H_{0}^{1}(\Omega)$ and $L^{p}(\Omega)(1<p<\infty)$ (P97 [1]). And, combining with $\|x\|_{X}=\|y\|_{X}=1$, we can get the constant $c=$ 1 , which contradicts the assumption $x \neq y$. Therefore, the Banach space $X$ is strictly convex.

For the reflexive, we need the following lemmas (see P63, P105 [2]).

Lemma 3. Let $X_{1}, \ldots, X_{n}$ be normed space. Then $X_{1} \oplus \cdots \oplus X_{n}$ is a Banach spaces if and only if each $X_{j}$ is a Banach space; furthermore, $X_{1} \oplus \cdots \oplus X_{n}$ is reflexive if and only if each $X_{j}$ is reflexive.

Lemma 4. Every closed subspace of a reflexive space is reflexive.

Therefore, setting a space $E=H_{0}^{1}(\Omega) \oplus L^{p}(\Omega)$ with the norm $\|\cdot\|_{X}=\|\cdot\|_{H_{0}^{1}(\Omega)}+\|\cdot\|_{L^{p}(\Omega)}$. It follows from Lemma 3 that $E$ is a reflexive Banach space. Obviously, our space $X=$ $H_{0}^{1}(\Omega) \cap L^{p}(\Omega)(1<p<\infty)$ with the norm $\|\cdot\|_{X}=$ $\|\cdot\|_{H_{0}^{1}(\Omega)}+\|\cdot\|_{L^{p}(\Omega)}$ can be seen as a closed subspace of $E$ by the embedded mapping $u \rightarrow(u, u)$ (denoting $(u, u)$ by $i(u)$ in the following). Thus, based on Lemma $4, X$ is a reflexive Banach space.

For the dual, we need the following lemma (see P91 [2]).

Lemma 5. Let $X_{1}, \ldots, X_{n}$ be normed spaces. Then there is an isometric isomorphism that identifies $\left(X_{1} \oplus \cdots \oplus X_{n}\right)^{*}$ with
$X_{1}^{*} \oplus \cdots \oplus X_{n}^{*}$, such that, if the element $y^{*}$ of $\left(X_{1} \oplus \cdots \oplus X_{n}\right)^{*}$ is identified with the element $x_{1}^{*}, \ldots, x_{n}^{*}$ of $X_{1}^{*} \oplus \cdots X_{n}^{*}$, then

$$
y^{*}\left(x_{1}, \ldots, x_{n}\right)=\sum_{j=1}^{n} x_{j}^{*} x_{j}
$$

whenever $\left(x_{1}, \ldots, x_{n}\right) \in X_{1} \oplus \cdots \oplus X_{n}$.

From the Lemma 5, we know that the dual space $E^{*}$ of $E=H_{0}^{1}(\Omega) \oplus L^{p}(\Omega)$ will be $E^{*}=H^{-1}(\Omega) \oplus L^{q}(\Omega)$ with $(1 / p)+(1 / q)=1$. And, if our space $X=H_{0}^{1}(\Omega) \cap L^{p}(\Omega)$ can be seen as a closed subspace of $E$, then, $X^{*} \subseteq H^{-1}(\Omega) \oplus L^{q}(\Omega)$ (in the sense of restriction). At the same time, from the HahnBanach theorem, we know that, for any $f \in X^{*}$, we can extend $f$ to be a bounded linear functional $\tilde{f}$ on $E$, such that

$$
\langle\tilde{f}, i(u)\rangle_{E^{*}, E}=\langle f, u\rangle_{X^{*}, X} \quad \forall u \in X .
$$

And, from (7), we have

$$
\langle\widetilde{f}, i(u)\rangle_{E^{*}, E}=\left\langle f_{1}, u\right\rangle_{H^{-1}(\Omega), H_{0}^{1}(\Omega)}+\left\langle f_{2}, u\right\rangle_{L^{q}(\Omega), L^{p}(\Omega)} .
$$

Hence,

$$
\begin{aligned}
\langle f, u\rangle_{X^{*}, X} & =\langle\tilde{f}, i(u)\rangle_{E^{*}, E} \\
& =\left\langle f_{1}, u\right\rangle_{H^{-1}(\Omega), H_{0}^{1}(\Omega)}+\left\langle f_{2}, u\right\rangle_{\left(L^{q} \Omega\right), L^{p}(\Omega)}
\end{aligned}
$$

which implies that $f \in E^{*}$. Therefore, $X^{*} \subset H^{-1}(\Omega) \oplus L^{q}(\Omega)$ and the proof of Theorem 2 is completely finished.

Also, for the property of weak converge in $X$, we have the following.

Lemma 6. If $u_{n} \rightarrow v$ in $X$ as $n \rightarrow \infty$, then

$$
\begin{gathered}
u_{n} \rightarrow v \quad \text { in } H_{0}^{1}(\Omega), \quad u_{n} \rightarrow v \quad \text { in } L^{p}(\Omega) \quad \text { as } n \rightarrow \infty \\
u_{n} \rightarrow v \text { in } L^{t}(\Omega) \quad \forall 2 \leqslant t<p \quad \text { as } n \rightarrow \infty
\end{gathered}
$$

Proof. In fact, for (11), from Theorem 2, we know that, for any $f \in X^{*}$, there exists $f_{1} \in H^{-1}(\Omega)$ and $f_{2} \in L^{q}(\Omega)$, such that

$$
\langle f, u\rangle_{X^{*}, X}=\left\langle f_{1}, u\right\rangle_{H^{-1}(\Omega), H_{0}^{1}(\Omega)}+\left\langle f_{2}, u\right\rangle_{L^{q}(\Omega), L^{p}(\Omega)} .
$$

Now, choosing $f_{2}=0$ in (13) (noting the fact that $H^{-1}(\Omega) \times$ $\left.\{0\} \subset X^{*}\right)$ and combining with $u_{n} \rightarrow v$ in $X$, we know that, for any $f_{1} \in H^{-1}(\Omega)$,

$$
\begin{aligned}
\left\langle f, u_{n}\right\rangle_{X^{*}, X} & =\left\langle f_{1}, u_{n}\right\rangle_{H^{-1}(\Omega), H_{0}^{1}(\Omega)}+\left\langle 0, u_{n}\right\rangle_{L^{q}(\Omega), L^{p}(\Omega)} \\
& =\left\langle f_{1}, u_{n}\right\rangle_{H^{-1}(\Omega), H_{0}^{1}(\Omega)} \longrightarrow\left\langle f_{1}, v\right\rangle_{H^{-1}(\Omega), H_{0}^{1}(\Omega)}
\end{aligned}
$$

which implies that $u_{n} \rightarrow v$ in $H_{0}^{1}(\Omega)$. Similarly, choosing $f_{1}=$ 0 in (13) (also noting the fact that $\{0\} \times L^{q}(\Omega) \subset X^{*}$ ), we can get $u_{n} \rightarrow v$ in $L^{p}(\Omega)$ and finish the proof of (11). 
And, for (12), from the interpolation inequality,

$$
\|u-v\|_{L^{t}(\Omega)} \leqslant\|u-v\|_{L^{2}(\Omega)}^{\theta}\|u-v\|_{L^{p}(\Omega)}^{1-\theta}
$$

and $H_{0}^{1}(\Omega) \hookrightarrow L^{2}(\Omega)$ with (11), it is easy to prove (12) and complete the proof of Lemma 6.

For the operators generated by (1), we have, for any $p>1$

Lemma 7. Both of operators $-\Delta: u \rightarrow-\Delta u$ from $H_{0}^{1}(\Omega)$ to $H^{-1}(\Omega)$ and $F: u \rightarrow|u|^{p-2} u$ from $L^{p}(\Omega)$ to $L^{p^{\prime}}(\Omega)=$ $L^{p /(p-1)}(\Omega)$ are bijective, where $\|u\|_{H_{0}^{1}}^{2}=\int_{\Omega} \nabla u \nabla u$.

Proof. First, for any $u \in H_{0}^{1}$, it follows that $-\Delta u \in H^{-1}$ from

$$
\langle-\Delta u, v\rangle_{H^{-1}, H_{0}^{1}}=\int_{\Omega} \nabla u \nabla v, \quad \forall v \in H_{0}^{1} .
$$

If $u \neq v \in H_{0}^{1}$, it follows from the maximum principle (see P179 Theorem 8.1 [3]) that $-\Delta u \neq-\Delta v \in H^{-1}$, which implies that it is an injection. Whereas, by Riesz's Lemma, we know that, for any $f \in H^{-1}$, there exists a $u \in H_{0}^{1}(\Omega)$, such that $\|f\|_{H^{-1}}=\|u\|_{H_{0}^{1}}$ and

$$
\langle f, v\rangle_{H^{-1}, H_{0}^{1}}=(u, v)_{H_{0}^{1}}=\int_{\Omega} \nabla u \nabla v=\int_{\Omega}-\Delta u v \quad \forall v \in H_{0}^{1}(\Omega)
$$

which implies that $f=-\Delta u$ and $\|-\Delta u\|_{H^{-1}}=\|f\|_{H^{-1}}=$ $\|u\|_{H_{0}^{1}}$. Hence $-\Delta: H_{0}^{1}(\Omega) \rightarrow H^{-1}(\Omega)$ is bijective (indeed, isometric).

Secondly, the map $F: u \rightarrow|u|^{p-2} u$ is clearly bounded, continuous, and also injective; namely, if $u \neq v \in L^{p}(\Omega)$, then $|u|^{p-2} u \neq|v|^{p-2} v \in L^{p^{\prime}}$, which can be obtained by the following inequality $\left\langle|u|^{p-2} u-|v|^{p-2} v, u-v\right\rangle \geqslant(1 / p)|u-v|^{p}$. For surjective, by applying the James Theorem in Banach space (see [4]) to the strictly convex space $L^{p}$ and $L^{p^{\prime}}$, for any $\|w\|_{L^{p^{\prime}}}=1$, there is only one unique supporting functional $\|u\|_{L^{p}}=1$, such that $\langle w, u\rangle=1$, which implies that $w=$ $|u|^{p-2} u$. So $F$ is bijective.

For the regularity of solution of (1), we have the following.

Lemma 8. Assuming $u_{0} \in X$ satisfies in the weak sense

$$
\begin{gathered}
-\Delta u+|u|^{r-2} u=f(x, u) \quad \text { in } \Omega \\
u=0 \quad \text { on } \partial \Omega
\end{gathered}
$$

then one has $u_{0} \in C^{1, \alpha}(\bar{\Omega})$, for all $\alpha<1$.

Proof. Indeed, we set the corresponding evolution equation

$$
\begin{gathered}
u_{t}-\Delta u+|u|^{r-2} u=f(x, u) \quad \text { in } \Omega \\
u=0 \quad \text { on } \partial \Omega
\end{gathered}
$$

and apply the same argument by the Moser iteration as Lemma 5.20 in [5], and, with the fact that the solution of (18) is the equilibrium point of (19), it is easy to show Lemma 8.

Now, we are in a position to prove Theorem 1.
Proof. Suppose the conclusion (3) does not hold. Then

$\forall \epsilon>0, \quad \exists v_{\epsilon} \in B_{\epsilon} \quad$ such that $\Phi\left(v_{\epsilon}\right)<\Phi(0)$

where $B_{\epsilon}=\left\{u \in X ;\|u\|_{X} \leqslant \epsilon\right\}$.

Claim 1. $\min _{B_{\epsilon}} \Phi$ is achieved at some point (still denoted by $\left.v_{\epsilon}\right)$.

Indeed, it is clear that there exists a constant $C$, such that $\|\Phi(u)\| \leqslant C$ for all $u \in B_{\epsilon}$. Hence, there exists a minimizing sequence $u_{n} \in B_{\epsilon}$, and there is by Lemma 6 a subsequence (also denoted by $u_{n}$ ) such that $u_{n} \rightarrow u$ in $H_{0}^{1}(\Omega), L^{p}(\Omega)$. Combining with the lower semicontinuity of norm, we have $\liminf _{n \rightarrow \infty}\left\|u_{n}\right\|_{H_{0}^{1}(\Omega)} \geqslant\|u\|_{H_{0}^{1}(\Omega)}$, $\liminf _{n \rightarrow \infty}\left\|u_{n}\right\| \geqslant\|u\|_{L^{p}(\Omega)}$, and $\lim _{n \rightarrow \infty} F\left(x, u_{n}\right) \rightarrow$ $F(x, u)$. Hence, $\liminf _{n \rightarrow \infty} \Phi\left(u_{n}\right) \geqslant \Phi(u)$ and Claim 1 is completely proved.

Now we will prove that $v_{\epsilon} \rightarrow 0$ in $C^{1}$, but (3) and (20) are contradictory (also see [6]). The corresponding Euler equation for $v_{\epsilon}$ involves a Lagrange multiplier $\mu_{\epsilon} \leqslant 0$; namely, $v_{\epsilon}$ satisfies

$$
\left\langle\Phi^{\prime}\left(v_{\epsilon}\right), \zeta\right\rangle_{X^{*}, X}=\mu_{\epsilon}\left\langle i\left(v_{\epsilon}\right), \zeta\right\rangle_{X^{*}, X}, \quad \forall \zeta \in X
$$

where $i\left(v_{\epsilon}\right)=-2 \Delta v_{\epsilon}+p\left|v_{\epsilon}\right|^{p-2} v_{\epsilon}$ due to Lemma 7 .

That is,

$$
\begin{array}{r}
\int_{\Omega} \nabla v_{\epsilon} \nabla \zeta+\int_{\Omega}\left|v_{\epsilon}\right|^{p-2} v_{\epsilon} \zeta-\int_{\Omega} f\left(x, v_{\epsilon}\right) \zeta \\
=2 \mu_{\epsilon} \int_{\Omega} \nabla v_{\epsilon} \nabla \zeta+\mu_{\epsilon} p \int_{\Omega}\left|v_{\epsilon}\right|^{p-2} v_{\epsilon} \zeta
\end{array}
$$

This means that

$$
-\left(1-2 \mu_{\epsilon}\right) \Delta v_{\epsilon}+\left(1-p \mu_{\epsilon}\right)\left|v_{\epsilon}\right|^{p-2} v_{\epsilon}=f\left(x, v_{\epsilon}\right) .
$$

Recalling that $\mu_{\epsilon} \leqslant 0$ and combining with Lemma 8 , one may bootstrap the bound $\left\|v_{\epsilon}\right\|_{X} \leqslant C$ to $\left\|v_{\epsilon}\right\|_{C^{1}} \leqslant C$ (independent of $\epsilon$ ), since $v_{\epsilon} \rightarrow 0$ in $X, v_{\epsilon} \rightarrow 0$ in $C^{1}$ (by Ascoli). This concludes the proof.

\section{Application of Theorem 1}

Next, we present a simple and useful application of Theorem 1.

Considering $\Phi$ in Theorem 1 with $f$, such that, for some constant $k$,

$$
f(x, u)+k u \text { is nondecreasing } u \text { for a.e. } x \text {. }
$$

Assume that there are $C(\bar{\Omega})$ sub- and supersolutions $\underline{u}$ and $\bar{u}$; that is, in the distribution sense,

$$
\begin{gathered}
-\Delta \underline{u}+|\underline{u}|^{p-2} \underline{u}-f(x, \underline{u}) \\
\leqslant 0 \leqslant-\Delta \bar{u}+|\bar{u}|^{p-2} \bar{u}-f(x, \bar{u}) \quad \text { in } \Omega \\
\underline{u} \leqslant 0 \leqslant \bar{u} \quad \text { on } \partial \Omega .
\end{gathered}
$$

Moreover, neither $\underline{u}$ nor $\bar{u}$ is a solution to (18). 
Theorem 9. Under the assumption (2) there is a solution $u_{0}$ to (18), $\underline{u}<u_{0}<\bar{u}$, such that, in addition, $u_{0}$ is a local minimum of $\Phi$ in $X$.

The proof relies on Theorem 1 as well as on the following.

Theorem 10. Let $\Omega$ be a bounded domain in $\mathbb{R}^{n}$ with smooth boundary $\partial \Omega$. Let $u \in L_{\mathrm{loc}}^{p-1}(\Omega)$ and assume that, for some $k \geqslant$ 0 , u satisfies

$$
\begin{gathered}
-\Delta u+|u|^{p-2} u+k u \geqslant 0 \quad \text { in } \Omega . \\
u \geqslant 0 \quad \text { on } \Omega
\end{gathered}
$$

Then either $u \equiv 0$ or there exists $\epsilon>0$, such that

$$
u(x) \geqslant \epsilon \operatorname{dist}(x, \partial \Omega) \quad \text { in } \Omega .
$$

Moreover, if $k$ is replaced by the nonnegative continuous function $c(x) \in C(\bar{\Omega})$, then the conclusion is also valid.

Proof. The measure $\mu=-\Delta u+|u|^{p-2} u+k u$ is nonnegative in $\Omega$. We may assume $u \neq \equiv$.

Case 1. Consider $\mu \equiv 0$. In this case, $u \in C^{\infty}(\Omega)$ by induction applies to Lemma 8:

$$
-\Delta u+|u|^{p-2} u+k u=0, \quad u \geqslant 0 \text { in } \Omega .
$$

Since $u \neq \equiv$, we have $u \geqslant \delta>0$ in some closed ball $B$ in $\Omega$. Let $\Omega_{j}$ be an expanding sequence of subdomains of $\Omega$ with smooth boundaries and $\bigcup_{j} \Omega_{j}=\Omega$; suppose $B \subset \Omega_{j}$, for all $j$. Let $h_{j}$ be the solution in $\Omega_{j} \backslash B$ of

$$
\begin{gathered}
-\Delta h_{j}+\left|h_{j}\right|^{p-2} h_{j}+k h_{j}=0, h_{j} \geqslant 0 \quad \text { in } \Omega_{j} \backslash B \\
h_{j}=\delta \quad \text { on } \partial B \\
h_{j}=0 \quad \text { on } \partial \Omega .
\end{gathered}
$$

In order to compare $u$ with $h_{j}$, we need the following comparison principle for the operator $L=-\Delta+|\cdot|^{p-2}$. defined in Lemma 7

Lemma 11. Let $u, v \in C^{1, \alpha}(\bar{\Omega})$ satisfy, for some $k \geqslant 0$,

$$
\begin{gathered}
L u+k u \geqslant L v+k v \quad \text { in } \Omega \\
u \geqslant v \quad \text { on } \partial \Omega ;
\end{gathered}
$$

then, $u \geqslant v$ in $\Omega$.

Proof. Indeed, setting

$$
L u-L v=-\Delta u+|u|^{p-2}-\left(-\Delta v+|v|^{p-2} v\right)
$$

and defining $w=u-v$, it is noted that the derivative expression $\left(|s|^{p-2} s\right)^{\prime}=(p-1)|s|^{p-2} \geqslant 0$. Then, by the mean value theorem, there is $\xi=\theta u+(1-\theta) v(0<\theta<1)$ satisfying

$$
\begin{gathered}
-\Delta w+(p-2)|\xi|^{p-2} w+k w \geqslant 0 \quad \text { in } \Omega \\
w \geqslant 0 \quad \text { on } \partial \Omega .
\end{gathered}
$$

Applying the weak maximum principle, Theorem 8.1 P179 [3] by choosing $c(x)=(p-2)|\xi|^{p-2}$, we know that $w \geqslant 0$ and complete the proof.

Since $u(x), h(x) \in C^{1, \alpha}\left(\overline{\Omega_{j} \backslash B}\right)$ in Lemma 8 , then, by the virtue of Lemma 11, $u \geqslant h_{j}$ in $\Omega_{j} \backslash B$. As $j \rightarrow \infty$, we find

$$
u \geqslant h \quad \text { in } \Omega \backslash B
$$

when $h$ solves

$$
\begin{gathered}
-\Delta h+|h|^{p-2} h+k h=0, \quad h \geqslant 0 \quad \text { in } \Omega \backslash B \\
h=\delta \quad \text { on } \partial B \\
h=0 \quad \text { on } \partial \Omega .
\end{gathered}
$$

By the Hopf lemma 3.4 P34 [3] with $c(x)=|h|^{p^{-2}}+k$, one obtains for some $\epsilon>0$

$$
h(x) \geqslant \epsilon \operatorname{dist}(x, \partial \Omega) \quad \text { in } \Omega \backslash B .
$$

The conclusion of Theorem 10 then follows directly.

Case 2. Consider $\mu \neq \equiv 0$. Let $\zeta \in C_{0}^{\infty}(\Omega)$ be a cutoff function, $0 \leqslant \zeta \leqslant 1$, such that $\zeta \mu \neq \equiv 0$. Let $v$ be the solution of

$$
\begin{gathered}
(L+k) v=\zeta \mu \quad \text { in } \Omega \\
v=0 \quad \text { on } \partial \Omega .
\end{gathered}
$$

Since $v$ is smooth outside a compact set $K \subset \Omega$, it follows and applies to the Hopf lemma as above for some $\epsilon>0$,

$$
v(x) \geqslant \epsilon \operatorname{dist}(x, \partial \Omega) \quad \text { in } \Omega \backslash B .
$$

The conclusion of Theorem 10 is a direct consequence of the following.

Claim 2. One has $u \geqslant v$ in $\Omega$.

Proof of Claim 2. Given any $\alpha>0$, we will prove that

$$
\bar{u}=u+\alpha \geqslant v \quad \text { in } \Omega .
$$

The claim then follows.

Note that $w=\bar{u}-v$ satisfies

$$
\begin{aligned}
(-\Delta+k) w+|\bar{u}|^{p-2} \bar{u}-|v|^{p-2} v & \\
= & (1-\zeta) \mu+|\bar{u}|^{p-2} \bar{u}-|u|^{p-2} u+k \alpha \geqslant 0 \quad \text { in } \Omega \\
& w \geqslant 0 \text { in } N_{\eta}=\{x \in \Omega ; \operatorname{dist}(x, \partial)<\eta\}
\end{aligned}
$$

provided $\eta$ is sufficiently small (depending on $\alpha$ ). The property (39) follows from the inequality $|\bar{u}|^{p-2} \bar{u}-|u|^{p-2} u \geqslant$ 0 , a.e. $x \in \Omega$, when $\bar{u}=u+\alpha>u$. The last property (40) follows from the fact that $v$ is smooth near $\partial \Omega$ and $v=0$ on $\partial \Omega$.

Let $\rho_{j}$ be a sequence of mollifiers with supp $\rho_{j} \subset B(0,1 / j)$ and set $w_{j}(x)=\int_{\Omega} \rho_{j}(x-y) w(y)$. 
Clearly $w_{j}$ is smooth, and, by (39) and the mean value theorem with $\xi=\theta \bar{u}+(1-\theta) v$, we have

$$
\left(-\Delta+k+(p-1)|\xi|^{p-2}\right) w_{j} \geqslant 0 \quad \text { in } \Omega \backslash \bar{N}_{1 / j} .
$$

On the other hand, we deduce from (40) that

$$
w_{j} \geqslant 0 \quad \text { on } \partial\left(\Omega \backslash \bar{N}_{1 / j}\right)
$$

provided $\eta>2 / j$. The maximum principle (Corollary $3.2 \mathrm{P} 33$ [3]) of choosing $c(x)=(p-1)|\xi|^{p-2} \geqslant 0$ implies that

$$
w_{j} \geqslant 0 \quad \text { in } \Omega \backslash \bar{N}_{1 / j}
$$

when $\eta>2 / j$. Passing to the limit as $j \rightarrow \infty$, we see that

$$
w \geqslant 0 \quad \text { in } \Omega
$$

which is the desired conclusion. The similar argument is also true as $k$ is replaced by the nonnegative continuous function $c(x) \in C(\bar{\Omega})$ and the proof of Theorem 10 is completely finished.

Now we are in a position to prove Theorem 9.

Proof of Theorem 9. On the basis of our above results, we can prove Theorem 9 by the similar argument as [7] and rewrite it here for the reader's convenience. We introduce an auxiliary function. Set

$$
\tilde{f}(x, s)= \begin{cases}f(x, \underline{u}(x)) & \text { if } s<\underline{u}(x) \\ f(x, s) & \text { if } \underline{u}(x) \leqslant s \leqslant \bar{u}(x) \\ f(x, \bar{u}(x)) & \text { if } s>\bar{u}(x)\end{cases}
$$

it is continuous in $s$. Then set

$$
\begin{gathered}
\widetilde{F}(x, u)=\int_{0}^{u} \tilde{f}(x, s) d s \\
\widetilde{\Phi}(u)=\frac{1}{2} \int_{\Omega}|\nabla u|^{2}+\frac{1}{p}|u|^{p}-\int_{\Omega} \widetilde{F}(x, u) .
\end{gathered}
$$

By the similar argument as Claim 1 , there is a minimum $u_{0} \epsilon$ $X$ satisfying

$$
-\Delta u_{0}+\left|u_{0}\right|^{p-2} u_{0}=\tilde{f}\left(x, u_{0}\right) \quad \text { in } \Omega .
$$

And, with Lemma 8 , we can get $u_{0} \in W^{2, p}$, for all $p<\infty$. We claim that $\underline{u} \leqslant u_{0} \leqslant \bar{u}$; we will just prove the first inequality. Indeed, we have

$$
L(\underline{u})-L\left(u_{0}\right) \leqslant f(x, \underline{u})-\widetilde{f}\left(x, u_{0}\right)
$$

and in particular

$$
L(\underline{u})-L\left(u_{0}\right) \leqslant 0 \quad \text { in } A=\left\{x \in \Omega ; u_{0}(x)<\underline{u}(x)\right\} .
$$

Since $\underline{u}-u_{0} \leqslant 0$ on $\partial A$, it follows from the comparison principle (i.e., Lemma 11) that $\underline{u}-u_{0} \leqslant 0$ in $A$. Therefore, $A=\emptyset$ and the claim is proved.
Returning to (48), we have

$$
\begin{aligned}
L(\underline{u}) & -L\left(u_{0}\right)+k\left(\underline{u}-u_{0}\right) \\
& \leqslant(f(x, \underline{u})+\underline{u})-\left(f\left(x, u_{0}\right)+k u_{0}\right) \leqslant 0 .
\end{aligned}
$$

Since $\underline{u}$ is not a solution, it follows from Theorem 10 that there is some $\epsilon>0$, such that

$$
\underline{u}(x)-u_{0}(x) \leqslant-\epsilon \operatorname{dist}(x, \partial \Omega), \quad \forall x \in \Omega .
$$

Similarly, for $\bar{u}$, we have

$$
\begin{array}{r}
\underline{u}(x)+\epsilon \operatorname{dist}(x, \partial \Omega) \leqslant u_{0}(x) \leqslant \bar{u}(x)-\epsilon \operatorname{dist}(x, \partial \Omega), \\
\forall x \in \Omega .
\end{array}
$$

It follows that, if $u \in C_{0}^{1}(\bar{\Omega})$ and $\left\|u-u_{0}\right\|_{C^{1}} \leqslant \epsilon$, then

$$
\underline{u} \leqslant u \leqslant \bar{u} \quad \text { in } \Omega \text {. }
$$

Finally, we apply the fact that $\widetilde{F}(x, u)-F(x, u)$ is a function of $x$ alone for $u \in[u(x), \bar{u}(x)]$. In particular, $\Phi(u)-\widetilde{\Phi}(u)$ is constant for $\left\|u-u_{0}\right\|_{C^{1}} \leqslant \epsilon$. Hence, $u_{0}$ is a local minimum of $\Phi$ in the $C^{1}$ topology (since it is a global minimum for $\widetilde{\Phi}$ ). So, from Theorem 1 , we know that $u_{0}$ is also a local minimum of $\Phi$ in the $X$ topology and the proof of Theorem 9 is finished.

\section{Conflict of Interests}

The author declares that there is no conflict of interests regarding the publication of this paper.

\section{Acknowledgments}

This paper is partially supported by the Science Foundation of Fujian province (2012J05002), Post-Doctor Foundation of China Grant (2011M501074), and the Innovation Foundation of Fujian Normal University (IRTL1206).

\section{References}

[1] H. Brezis, Functional Analysis, Sobolev Spaces and Partial Differential Equations, Universitext, Springer, New York, New York, NY, USA, 2011.

[2] R. E. Megginson, An Introduction to Banach Space Theory, vol. 183 of Graduate Texts in Mathematics, Springer, New York, NY, USA, 1998.

[3] D. Gilbarg and N. S. Trudinger, Elliptic Partial Differential Equations of Second Order, Classics in Mathematics, Springer, Berlin, Germany, 2001.

[4] B. Beauzamy, Introduction to Banach Spaces and Their Geometry, vol. 68 of North-Holland Mathematics Studies, NorthHolland, Amsterdam, The Netherlands, 1982.

[5] C.-K. Zhong, M.-H. Yang, and C.-Y. Sun, "The existence of global attractors for the norm-to-weak continuous semigroup and application to the nonlinear reaction-diffusion equations," Journal of Differential Equations, vol. 223, no. 2, pp. 367-399, 2006. 
[6] D. G. de Figueiredo, "On the existence of multiple ordered solutions of nonlinear eigenvalue problems," Nonlinear Analysis: Theory, Methods \& Applications, vol. 11, no. 4, pp. 481-492, 1987.

[7] H. Brezis and L. Nirenberg, " $H^{1}$ versus $C^{1}$ local minimizers," Comptes Rendus de l'Académie des Sciences. Série I. Mathématique, vol. 317, no. 5, pp. 465-472, 1993. 


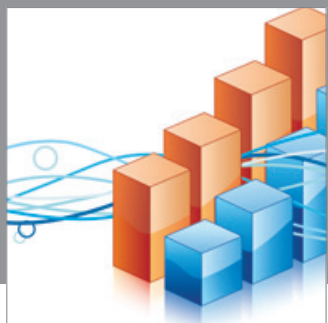

Advances in

Operations Research

mansans

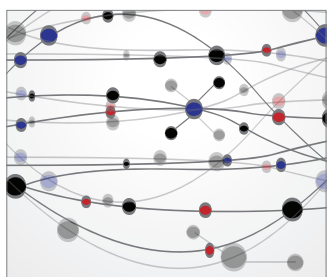

The Scientific World Journal
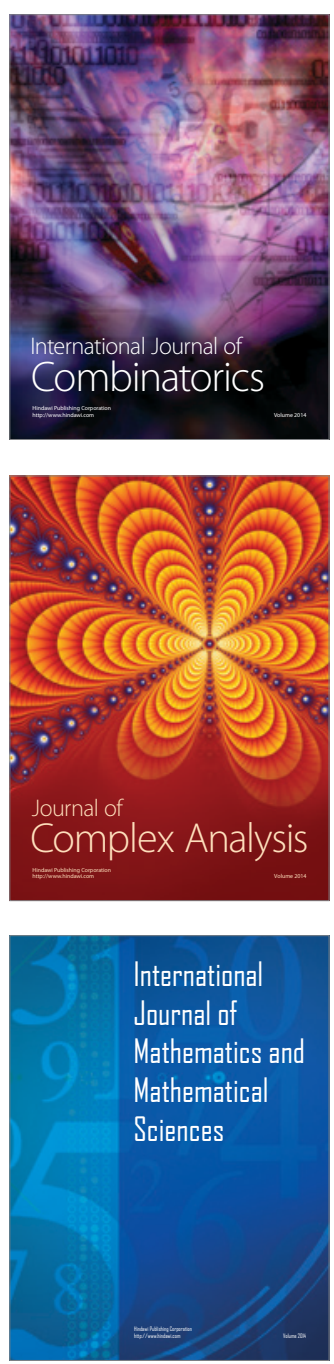
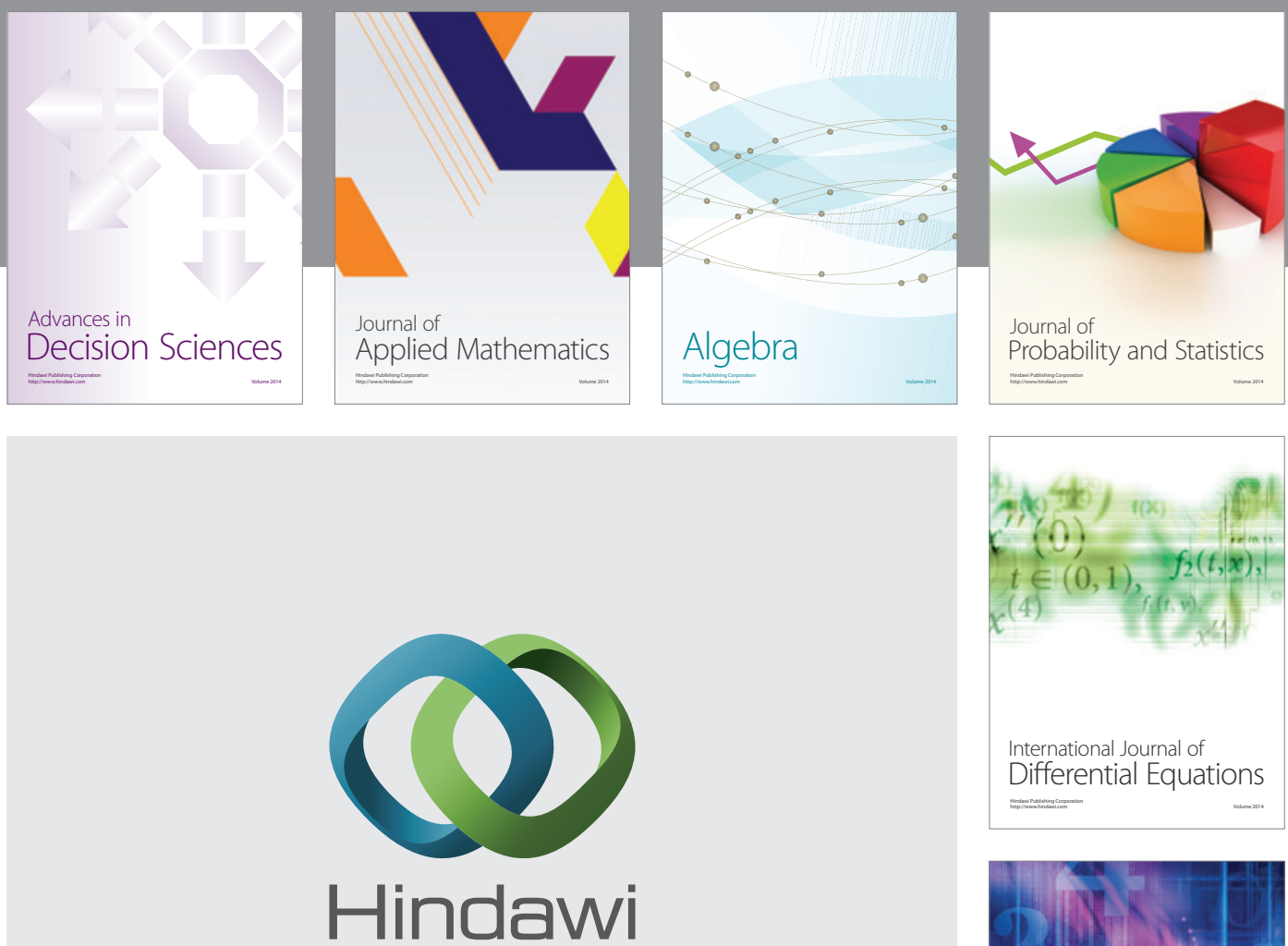

Submit your manuscripts at http://www.hindawi.com
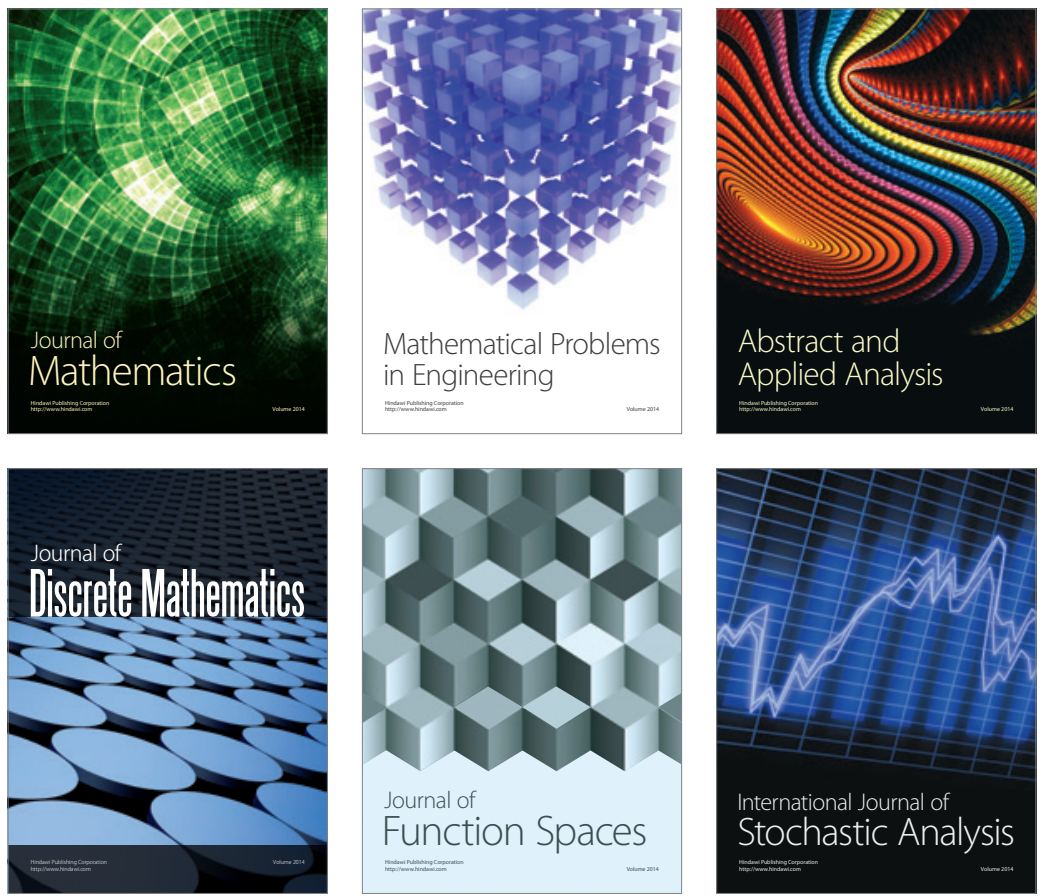

Journal of

Function Spaces

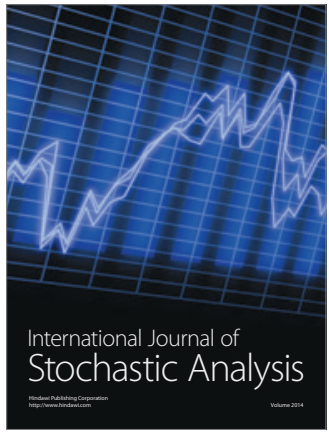

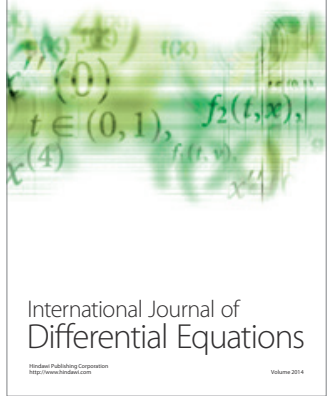
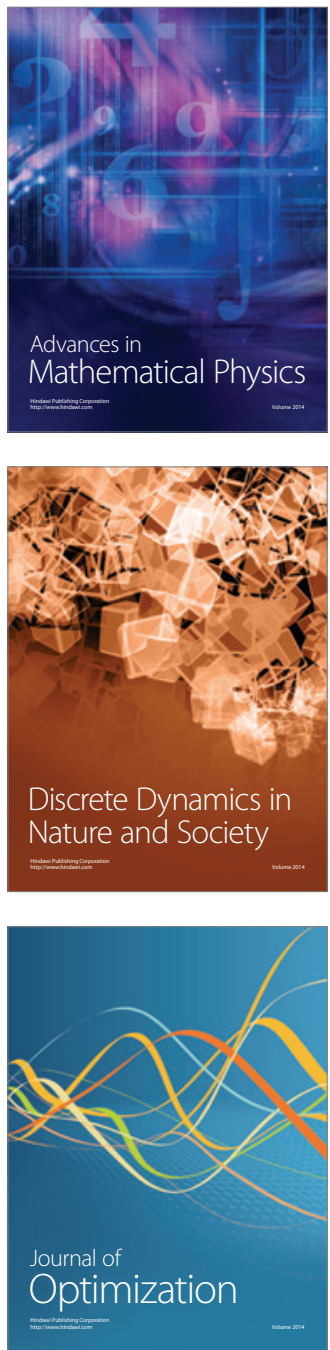